\title{
VERIFICATION OF THE TENFOLD ASSIGNMENT OF THE BARYON RESONANCES*
}

\author{
S. Meshkov ${ }^{\dagger}$
}

National Bureau of Standards, Washington, D. C。

\section{A. Levinson}

Department of Nuclear Physics, The Weizmann Institute of Science, Rehovoth, Israel

and

H. J. Lipkin $\ddagger$

Department of Physics, University of Illinois, Urbana, Illinois

(Received 18 February 1963)

One of the more attractive aspects of the octet model $^{1}$ or "eightfold way" has been its ability to accomodate the $N^{*}, Y_{1}^{*}$, and $\Xi^{*}$ resonances in one isostrangeness multiplet, the (30). ${ }^{2}$ The (30)

representation of $\mathrm{SU}_{3}$ is ten-dimensional as shown in Fig. 1. For this representation, the Okubo unitary mass formula ${ }^{2,3}$

$$
m=m_{0}\left\{1+a Y+b\left[T(T+1)-\frac{1}{4} Y^{2}\right]\right\}
$$

reduces to

$$
m=m_{0}{ }^{\prime}\left(1+a^{\prime} Y\right) .
$$

the $Y_{1}{ }^{*}(1385 \mathrm{MeV})$ are known, the mass of the $\Xi^{*}$ is predicted to be $1532 \mathrm{MeV}$. This predicted mass agrees amazingly well with the experimentally determined value of $1535^{4} \mathrm{MeV}$ giving strong support for the validity of the assignment of the baryon resonances to the (30) representation.

The present work proposes experimental tests for the validity of these assignments by examining the processes

meson + baryon $\rightarrow$ baryon resonance

Thus once the masses of the $N^{*}(1238 \mathrm{MeV})$ and

$$
\text { + vector meson, }
$$

a. Axis 1 perspective.

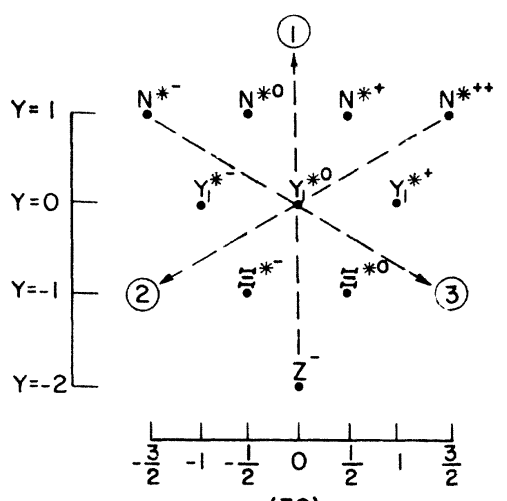

(30)

b. Axis 2 perspective.

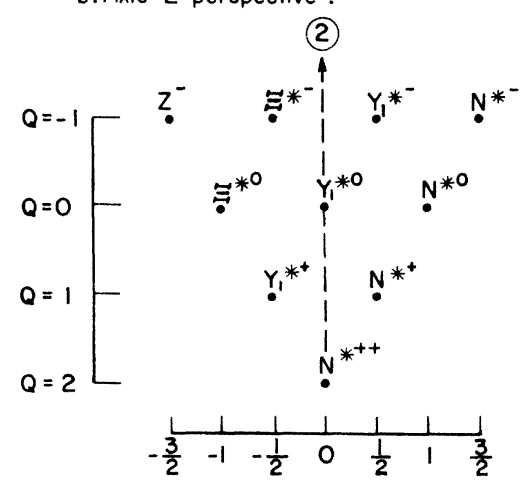

(30)

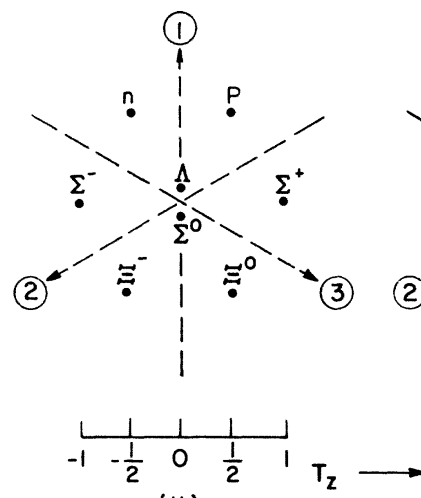

(II)

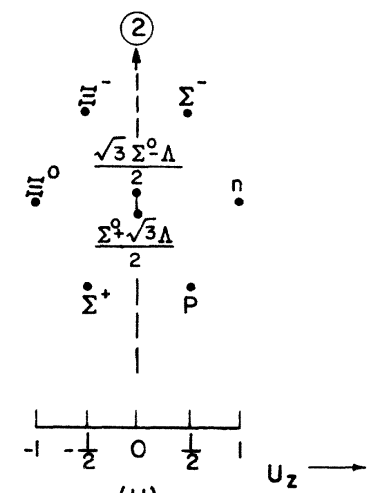

(11)
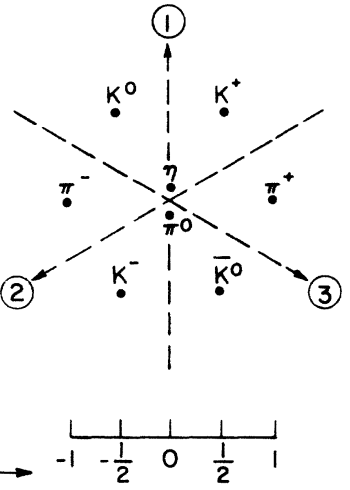

(II)

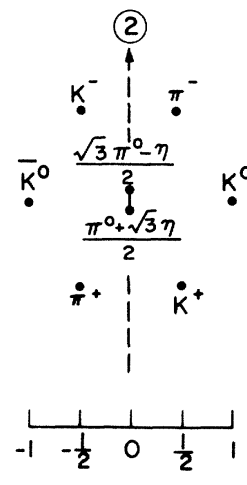

(II)

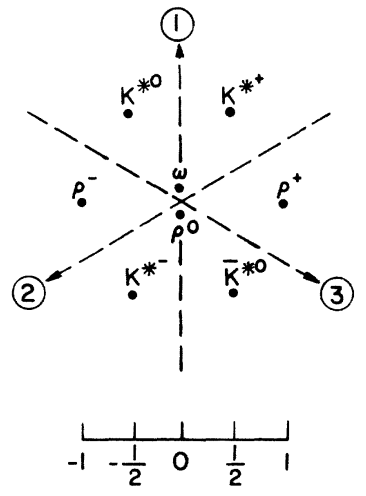

(II)

FIG. 1. Representation diagrams of the group $\mathrm{SU}_{3}$. The (30) baryon-resonance representation diagram and the (11) baryon, meson, and vector-meson representation diagrams are displayed. 
meson + baryon $\rightarrow$ baryon resonance + meson. $(3 \mathrm{~b})$

The method used has been described in detail ${ }^{5,6}$ for the processes

$$
\text { meson }+ \text { baryon } \rightarrow \text { meson }+ \text { baryon }
$$

according to both the Sakata and octet models.

The meson $(1,1)$ symmetry and the baryon $(1,1)$ symmetry can couple together to make product symmetries (22), (11), (11), (30), (03), and (00). Each product symmetry defines a channel through which the reaction can proceed. In a similar fashion the right-hand sides of Eqs. (3a) and (3b) are described by coupling the baryon-resonance (30) symmetry and either the vector-meson or meson (11) symmetries to produce symmetries (41), (30), (22), and (11). One can introduce the energy and angle dependent amplitudes $A^{(\lambda, \mu)}$ which are diagonal elements of the $S$ matrix, and in terms of which one obtains expressions for the amplitude for processes (3a) and (3b). Only those symmetries common to the left and right sides of (3a) and ( $3 b)$ contribute to the amplitude, i.e., (22), (30), and two (11)'s. The scattering amplitudes have been calculated from the overlaps of the meson-baryon functions (from which the results of reference 6 were obtained) with the functions corresponding to the product $(30) \times(11)$. The latter functions have been obtained by combining the tables of Rashid ${ }^{7}$ with the appropriate Clebsch-Gordan coefficients.

The following relations and equalities are then seen to hold among the scattering amplitudes:

$$
\begin{aligned}
\left(\pi^{-} p \mid Y_{1}{ }^{-} K^{*+}\right)= & \left(K^{-} p \mid \Xi^{*-} K^{*+}\right)=-\left(K^{-} p \mid Y_{1}{ }^{*-} \rho^{+}\right) \\
& =-(1 / \sqrt{3})\left(\pi^{-} p \mid N^{*-} \rho^{+}\right) .
\end{aligned}
$$

Alternatively $K^{*+}$ and $\rho^{+}$may be replaced by their meson analogs $K^{+}$and $\pi^{+}$to yield

$$
\begin{aligned}
\left(\pi^{-} p \mid Y_{1}{ }^{-} K^{+}\right) & =\left(K^{-} p \mid \Xi^{*-} K^{+}\right)=-\left(K^{-} p \mid Y_{1}{ }^{*-} \pi^{+}\right) \\
& =-(1 / \sqrt{3})\left(\pi^{-} p \mid N^{*-} \pi^{+}\right) .
\end{aligned}
$$

As a result of (5a) and (5b), definite predictions about the equalities and ratios of cross sections and polarizations are made, subject to the provisos ${ }^{5,6}$ of large enough incident energies and momentum transfer. The experimental angular distributions for the reactions $\left(\pi^{-} p \mid Y_{1}{ }^{*} K^{+}\right)^{8}$ and $\left(K^{-} p \mid Y_{1}{ }^{*-} \pi^{+}\right)^{9}$ show similar behavior, i.e., peaking of the $Y_{1}{ }^{*-}$ in the backward direction. This similarity of the angular distributions is in accord with the relations (5b). Backward peaking for the $\Xi^{*-}$ and the $N^{*-}$ in the remaining processes of $(5 \mathrm{~b})$ is then predicted.
The relations (5a) and ( $5 b)$ can be derived in a much more transparent fashion by consideration of a $\mathrm{U}_{2}$ subgroup of $\mathrm{U}_{3}$ different from isotopic spin. The usual weight-diagram representations of the $U_{3}$ multiplets are given in terms of isospin and hypercharge and are shown in Fig. 1(a). In these diagrams $U_{2}$ transformations perpendicular to axis one correspond to the transformations of isospin. However, $\mathrm{U}_{2}$ transformations perpendicular to axes two or three are also symmetry operations of $U_{3}$. We are particularly interested in the transformations perpendicular to axis two and have redrawn the weight diagrams with this axis vertical in Fig. 1(b). Members of the same $U_{2}$ multiplets in this scheme all have the same charge and are on the same horizontal line in Fig. 1(b). We shall call the $U_{2}$ transformations within these multiplets $U$-spin transformations, and shall therefore have multiplets of $U=\frac{3}{2}, 1, \frac{1}{2}, 0$ for the (30) representation and $U=\frac{1}{2}, 1,0, \frac{1}{2}$ for the (11) representation. The components of the $U$ multiplets are labeled by $U_{z}$ as indicated in Fig. 1(b).

In the (11) diagram there is a charge zero $U$ spin triplet and $U$-spin singlet. For the vectormeson octet the $U$-spin triplet with $U_{z}=0$ is

$$
M_{1}=\frac{1}{2}\left(\rho^{0}+\sqrt{3} \omega\right)
$$

The $U$-spin singlet is

$$
M_{0}=\frac{1}{2}\left(\sqrt{3} \rho^{0}-\omega\right) .
$$

The physical $\rho^{0}$ and $\omega$ vector mesons are then given by

$$
\begin{aligned}
\rho^{0} & =\frac{1}{2}\left(M_{1}+\sqrt{3} M_{0}\right), \\
\omega & =\frac{1}{2}\left(\sqrt{3} M_{1}-M_{0}\right) .
\end{aligned}
$$

Identical transformations hold for the $\pi^{0}$ and $\eta$ mesons and for the $\Sigma^{0}$ and $\Lambda$ particles.

Let us now consider the $\pi^{-} p$ amplitudes of Eqs. (5). As may be seen from Fig. 1(b), the $\pi^{-} p$ system has $U_{z}=1, U=1$. The processes

$$
\pi^{-} p \rightarrow N^{*-}+\rho^{+}
$$

and

$$
\pi^{-} p \rightarrow Y_{1}^{*-}+K^{*+}
$$

are then determined only by a single $U=1$ amplitude, $T$. The branching ratio of these two processes is given solely by the Clebsch-Gordan coefficients, $\left(U_{1} U_{2} M_{1} M_{2} \mid U M\right)$ for the allowed couplings of the members of the $U=\frac{3}{2}$ multiplet (which contains $\mathrm{N}^{*^{-}}$and $\mathrm{Y}^{*^{-}}$) with the $U=\frac{1}{2}$ 
multiplet (which contains $\rho^{+}$and $K^{*+}$ ). It is

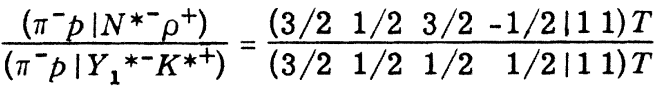

$$
\begin{aligned}
& =\frac{\frac{1}{2} \sqrt{3} T}{-\frac{1}{2} T}=-\sqrt{3} \text {. }
\end{aligned}
$$

The processes

$$
K^{-} p \rightarrow Y_{1}^{*-}+\rho^{+}
$$

and

$$
K^{-} p \rightarrow \Xi^{*-}+K^{*+}
$$

are similarly analyzed in terms of a single $U=1$ amplitude. $K^{-} p$ can couple to $U=0$ and 1 , whereas $Y_{1}{ }^{*-} \rho^{+}$and $\Xi^{*-} K^{*+}$ each may couple to $U=1$ and 2. Therefore, once again only the same $U$ $=1$ amplitude, $T$, occurs.

The scattering amplitudes for the processes (10) then have the ratio

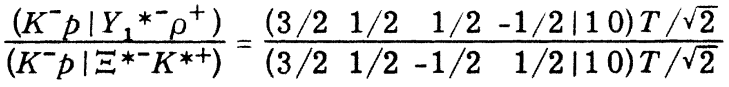

$$
\begin{aligned}
& =\frac{T / 2}{-T / 2}=-1 \text {. }
\end{aligned}
$$

Thus the relations (5) follow directly from the $U$-spin transformation.

In addition, other relations not as simple as those of (5) may be easily obtained using $U$-spin. Consider the amplitudes for the three processes initiated by $\pi^{-} p$, a pure $U=1$ channel:

$$
\begin{aligned}
& \left(\pi^{-} p \mid N^{* 0}+\rho^{0}\right), \\
& \left(\pi^{-} p \mid N^{* 0}+\omega\right), \\
& \left(\pi^{-} p \mid Y_{1}{ }^{0}+K^{* 0}\right) .
\end{aligned}
$$

The amplitudes in (12) are determined by two independent $U=1$ amplitudes. To define them let

$$
\begin{aligned}
& X=\left(\Xi^{* 0}, Y_{1}{ }^{* 0}, N^{* 0}\right), \\
& Y=\left(\bar{K}^{* 0}, \frac{1}{2}\left(\rho^{0}+\sqrt{3} \omega\right), K^{* 0}\right),
\end{aligned}
$$

and

$$
M_{0}=\frac{1}{2}\left(\sqrt{3} \rho^{0}-\omega\right)
$$

be the three $U$-spin multiplets of interest. Let $A$ and $B$ be the $U$-spin triplet amplitudes leading to $(X Y)$ and $\left(X M_{0}\right)$ triplet combinations. We will also need the $U$-spin singlet amplitude $C$ leading to $(X Y)$ in the singlet state.

The processes (12) yield the three relations in two parameters

$$
\begin{aligned}
& \left(\pi^{-} p \mid N^{* 0} \rho^{0}\right)=A / 2 \sqrt{2}+(\sqrt{3} / 2) B, \\
& \left(\pi^{-} p \mid N^{* 0} \omega\right)=\left(\sqrt{3} / 2^{\sqrt{2}}\right) A-\frac{1}{2} B,
\end{aligned}
$$

$$
\left(\pi^{-} p \mid Y_{1}{ }^{* 0} K^{* 0}\right)=-A / \sqrt{2} \text {. }
$$

A similar analysis of the four $K^{-} p$ amplitudes yields

$$
\begin{aligned}
& \left(K^{-} p \mid N^{* 0} \bar{K}^{* 0}\right)=\frac{1}{2} A-C / \sqrt{6}, \\
& \left(K^{-} p \mid \Xi{ }^{* 0} K^{* 0}\right)=-\frac{1}{2} A-C / \sqrt{6} \text {, } \\
& \left(K^{-} p \mid Y_{1}{ }^{* 0} \rho^{0}\right)=C / 2^{\sqrt{6}}+3 B / 2^{\sqrt{6}} \text {, } \\
& \left(K^{-} p \mid Y_{1}{ }^{* 0} \omega\right)=C / 2^{\sqrt{2}}-B / 2^{\sqrt{2}} \text {. }
\end{aligned}
$$

From the amplitudes given above, the following relations between differential cross sections hold:

$$
\begin{aligned}
\sigma(15 c)+ & \sigma(15 d)+\sigma(14 c) \\
& =\frac{1}{2}[\sigma(15 a)+\sigma(15 b)+\sigma(14 a)+\sigma(14 b)] .
\end{aligned}
$$

The seven processes (14) and (15) are seen to depend on only three independent amplitudes.

As pointed out before, any results obtained involving the vector-meson octet also hold when the vector mesons are replaced by their meson analogs.

We are indebted to George Snow for his encouragement and suggestions and to Gideon Alexander, Manoj Banerjee, and Gaurang Yodh for very useful discussions.

${ }^{*}$ The research reported in this document has been sponsored in part by the Office of Scientific Research, Office of Air Research, through the European Office, Aerospace Research, U. S. Air Force.

tWork done in part while a visitor at the Weizmann Institute of Science, Rehovoth, Israel.

$\ddagger$ On leave from the Weizmann Institute of Science, Rehovoth, Israel.

${ }^{1} \mathrm{Y}$. Ne'eman, Nucl. Phys. 26, 222 (1961); M. GellMann, California Institute of Technology Report CTSL20 (unpublished).

${ }^{2}$ S. L. Glashow and J. J. Sakurai, Nuovo Cimento 25 , 337 (1962); S. L. Glashow and J. J. Sakurai, Nuovo Cimento 26, 622 (1962); M. Gell-Mann, Proceedings of the International Conference on High-Energy Nuclear Physics, Geneva, 1962 (CERN Scientific Information Service, Geneva, Switzerland, 1962).

${ }^{3}$ S. Okubo, Progr. Theoret Phys. (Kyoto) 27, 949 (1962).

${ }^{4}$ G. M. Pjerrou, D. J. Prowse, P. Schlein, W. E. Slater, D. H. Stork, and H. K. Ticho, Phys. Rev. Letters 9 , 114 (1962); L. Bertanza, V. Brisson, P. L. Connolly, E. L. Hart, I. S. Mittra, G. C. Moneti, R. R. Rau, N. P. Samios, I. O. Skillicorn, S. S. Yamamoto, M. Goldberg, L. Gray, J. Leitner, S. Lichtman, and J. Westgard, Phys. Rev. Letters 9, 180 (1962).

${ }^{5}$ C. A. Levinson, H. J. Lipkin, and S. Meshkov, Nuovo Cimento $\underline{23}, 236$ (1962).

${ }^{6}$ C. A. Levinson, H. J. Lipkin, and S. Meshkov, Phys. Letters 1,44 (1962). 
${ }^{7}$ M. A. Rashid, Nuovo Cimento 26, 118 (1962).

${ }^{8}$ A. R. Erwin, R. H. March, and W. D. Walker, Nuovo Cimento 24, 237 (1962).
${ }^{9}$ R. P. Ely, Sun-Yiu Fung, George Gidal, Yu-Li Pan, Wilson M. Powell, and Howard S. White, Phys. Rev. Letters $\underline{7}, 461$ (1961).

\section{GAMMA RAYS AT HIGH ALTITUDES*}

J. G. Duthie, E. M. Hafner, and M. F. Kaplon

Department of Physics and Astronomy, University of Rochester, Rochester, New York

and

\section{G. G. Fazio}

Smithsonian Astrophysical Observatory, Cambridge, Massachusetts

(Received 21 February 1963)

We have carried out four high-altitude balloon flights in an investigation of primary gamma radiation. Our detector ${ }^{1}$ contained, as its principal element, a Cherenkov counter so designed as to yield simultaneous data on charged particles and gamma rays in wide and narrow cones of sensitivity. The effective opening of the wide cone was approximately $60^{\circ}$ in the first two flights and $30^{\circ}$ in the later flights; the opening of the narrow cone was $10^{\circ}$ in all of the experiments. Our system had a sensitive area of $100 \mathrm{~cm}^{2}$, a gammaray energy threshold of approximately $60 \mathrm{MeV}$, and a photon conversion efficiency of $21 \%$. The charged-particle channels were sensitive to all relativistic $(\beta>0.7)$ charged particles. The instrument was flown at $40^{\circ}$ geomagnetic latitude with its axis oriented to zenith at all times.

Table I summarizes the conditions of our flights and lists the data channels that we believe to have been operating correctly. In only one flight (April 1962) did we obtain trouble-free data in all four of the available channels. Electronic and mechanical difficulties have obscured five of the 16 possible sets of data that the series of flights could have produced. In addition, the 60degree gamma channel in the first flight (July 1961) could only be read from the record as the difference between two rapidly counting charged channels. The conclusions of this report are based on the remaining ten sets of reliable data.

Figure 1 shows the high-altitude response of the instrument to charged particles and gamma rays in each of our three geometries. The chargedparticle maximum counting rate occurs at about 130 millibars, the gamma-ray maximum at 180 millibars. Two of the gamma-ray curves show the effect of removing a lead converter $\left(1.9 \mathrm{~g} / \mathrm{cm}^{2}\right)$ in which electron pairs are produced. Our timers were set to give periodic "lead-out" data for 40 minutes of each four hours of flight. The gamma channel rates with lead out are consistently proportional to charged-particle rates in the same geometry. We are therefore able to make an accurate background subtraction at all times.

The altitude dependence of our 30-degree gamma data in the top two percent of atmosphere is shown in Fig. 2. In addition to making the "lead-out" subtraction described above, we have corrected these data for changes in geomagnetic latitude during the two flights. We make the correction by assuming that the extrapolated gamma count is independent of latitude while the slope of the curve is proportional to the primary charged flux. It is only after this correction is made that the two flights are in good agreement.

Also shown in Fig. 2 are three points obtained in a similar experiment by Cline. ${ }^{2}$ Using our known geometry and efficiency, we have converted

Table I. Experimental conditions.

\begin{tabular}{|c|c|c|c|c|c|c|c|c|}
\hline \multirow[b]{2}{*}{ Date } & \multirow[b]{2}{*}{ Max altitude } & \multirow{2}{*}{$\begin{array}{l}\text { Float } \\
\text { time }\end{array}$} & \multicolumn{3}{|c|}{ Charged } & \multicolumn{3}{|c|}{ Gamma } \\
\hline & & & $10^{\circ}$ & $30^{\circ}$ & $60^{\circ}$ & $10^{\circ}$ & $30^{\circ}$ & $60^{\circ}$ \\
\hline 26 July 1961 & $4.4 \mathrm{mb}$ & $8.8 \mathrm{~h}$ & $\times$ & & $x$ & & & $x$ \\
\hline 25 April 1962 & 5.4 & 7.0 & $x$ & & $x$ & $x$ & & $\times$ \\
\hline 28 September 1962 & 3.9 & 7.1 & \multicolumn{3}{|c|}{$x$} & \multicolumn{3}{|c|}{$x$} \\
\hline 4 October 1962 & 4.0 & 15.3 & \multicolumn{3}{|c|}{$\times$} & \multicolumn{3}{|c|}{$x$} \\
\hline
\end{tabular}

